Yüzüncü Y1l Üniversitesi
Fen Bilimleri Enstitüsü Dergisi
http://dergipark.gov.tr/yyufbed

Araştırma Makalesi

\title{
Modülüs Fonksiyon Yardımı ile Tanımlanan İnvaryant Yakınsak Dizi Uzaylarının Topolojik Özellikleri
}

\section{Hasan KARA ${ }^{1}$, Dinçer ATASOY*2}

${ }^{1}$ Iğdır Üniversitesi, Fen-Edebiyat Fakültesi, Matematik Bölümü, Iğdır, Türkiye

${ }^{2}$ Iğdır Üniversitesi, Iğdır Meslek Yüksekokulu, Finans-Bankacılık ve Sigortacılık Bölümü, Iğdır, Türkiye

Hasan KARA, ORCID No: 0000-0001-9828-9006, Dinçer ATASOY, ORCID No: 0000-0003-0389-1059

*Sorumlu yazar e-posta: dincer.atasoy@igdir.edu.tr

\section{Makale Bilgileri}

Geliş: 31.05 .2021

Kabul: 28.06.2021

Yayınlanma Ağustos 2021

DOI: $10.53433 /$ yyufbed.945323

\section{Anahtar Kelimeler}

Dizi uzaylarının topolojik

özellikleri,

İnvaryant yakınsak dizi,

Modülüs fonsiyonu
Öz: Bu çalışmada Modülüs fonksiyon yardımı ile tanımlanan invaryant yakınsak dizi uzayları tanımlanarak aralarında bazı kapsam bağıntıları kuruldu. $\left[\omega_{\sigma}(f)\right], \bar{\omega}_{\sigma}(f)$ ve $\overline{\bar{\omega}}_{\sigma}(f)$ uzayları $\left[\omega_{\sigma}(f)(p)\right], \bar{\omega}_{\sigma}(f)(p)$ ve $\overline{\bar{\omega}}_{\sigma}(f)(p)$ uzaylarına genişletildi. Genelleştirilen bu dizi uzaylarının topolojik özellikleri incelendi.

\section{Topological Properties of Invariant Convergent Sequences Defined with the Help of a} Modulus Function

\section{Article Info}

Recieved: 31.05.2021

Accepted: 28.06.2021

Published August 2021

DOI: $10.53433 /$ yyufbed.945323

\section{Keywords}

Topological properties of

sequence spaces,

Invariant convergent sequence,

Modulus function

\begin{abstract}
In this study, invariant convergent sequence spaces defined with the help of the Modulus function were defined and some scope relations were established beyween them. Spaces of $\left[\omega_{\sigma}(f)\right], \bar{\omega}_{\sigma}(f)$ and $\overline{\bar{\omega}}_{\sigma}(f)$ is extended to $\left[\omega_{\sigma}(f)(p)\right], \bar{\omega}_{\sigma}(f)(p)$ and $\overline{\bar{\omega}}_{\sigma}(f)(p)$ spaces. Topological properties of generalized sequence spaces are studied.
\end{abstract}




\section{Giriş}

$\mathrm{Bu}$ çalışmada modülüs fonksiyon yardımı ile tanımlanan invaryant yakınsak dizi uzayları tanımlanmıştır. $\left[\omega_{\sigma}(f)\right], \bar{\omega}_{\sigma}(f)$ ve $\overline{\bar{\omega}}_{\sigma}(\mathrm{f})$ uzayları $\left[\omega_{\sigma}(\mathrm{f})(\mathrm{p})\right], \bar{\omega}_{\sigma}(\mathrm{f})(\mathrm{p})$ ve $\overline{\bar{\omega}}_{\sigma}(\mathrm{f})(\mathrm{p})$ dizi uzaylarına genişletilmişir. Lorentz (1948), Savaş (2018), Rafeiro ve ark. (2018) ve Oğur (2020) tarafindan çeşitli yönleri ile çalışmalar yapılmıştır. Bu çalışmalar kapsamında daha genel dizi uzaylarının bazı topolojik özellikleri incelenmiştir.

\section{Tanım 1.}

$X$ bir lineer uzayı üzerinde bir $g: X \rightarrow \mathbb{R}$ bir fonksiyonu aşağıdaki özellikleri sağlıyorsa $g$ fonksiyonuna $X$ üzerinde bir paranorm ve $(X . g)^{\prime}$ ye de bir paranormlu uzay denir (Lorentz, 1948).

P1. $g(\theta)=0$

P2. $g(x)=g(-x)$

P3. $g(x+y) \leq g(x)+g(y)$

P4. $\lambda \rightarrow \lambda_{0}, x \rightarrow x_{0}$ olması , $\lambda x \rightarrow \lambda_{0} x_{0}$ olmasinı gerektirir.

\section{Tanım 2.} takdirde

$P=\left(P_{m}\right)$ her $m$ için $P_{m}>0$ ve Sup $P_{m}=H<\infty$ olacak şekilde reel sayılar dizisi olsun. $\mathrm{Bu}$

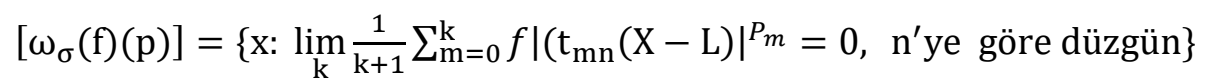

$\bar{\omega}_{\sigma}(\mathrm{f})(\mathrm{p})=\left\{\mathrm{x}: \sum_{\mathrm{n}=0}^{\infty} \mathrm{f}\left(\left|\Psi_{\mathrm{mn}}(\mathrm{x})-\mathrm{t}_{\mathrm{m}-1, \mathrm{n}}(\mathrm{x})\right|\right)^{P_{m}}\right), \mathrm{n}^{\prime}$ ye göre düzgün yakınsak $\}$

$\overline{\bar{\omega}}_{\sigma}(\mathrm{f})(\mathrm{p})=\left\{\mathrm{x}: \operatorname{Sup}_{\mathrm{n}} \sum_{\mathrm{m}=0}^{\infty} \mathrm{f}\left(\left|\Psi_{\mathrm{mn}}(\mathrm{x})-\Psi_{\mathrm{m}-1, \mathrm{n}}(\mathrm{x})\right|^{P_{m}}\right)<\infty\right\}$ dur.

$\forall m i \operatorname{çin}\left(P_{m}\right)=P$ olduğunda $\left[\omega_{\sigma \mathrm{p}}(\mathrm{f})\right], \bar{\omega}_{\sigma \mathrm{p}}(\mathrm{f})$ ve $\overline{\bar{\omega}}_{\sigma \mathrm{p}}(\mathrm{f})$ uzaylarına eşit olur.

Ayrıca $\sigma_{(n)}=n+1$ olduğunda, bu uzaylar $\left[\omega_{\sigma}(\mathrm{f})\right], \bar{\omega}_{\sigma}(\mathrm{f})$ ve $\overline{\bar{\omega}}_{\sigma}(\mathrm{f})$ dizi uzaylarına indirgenir. (Sahoo, 1992).

Şimdi tanımlanan bu uzaylar ile ilgili özellikleri verelim.

\section{Teorem 1.}

$\left[\omega_{\sigma}(f)(p)\right], \bar{\omega}_{\sigma}(f)(p)$ ve $\overline{\bar{\omega}}_{\sigma}(f)(p)$ kümeleri $\mathbb{C}$ üzerinde lineer uzaylardır.

\section{İspat.}

$\left[\omega_{\sigma}(f)(p)\right], \bar{\omega}_{\sigma}(f)(p)$ ve $\overline{\bar{\omega}}_{\sigma}(f)(p)$ kümeleri $\mathbb{C}$ kümesinin alt kümeleridir.

$\left[\omega_{\sigma}(f)(p)\right]$ kümesinin $\mathbb{C}$ üzerinde lineer uzay olduğunu göstereceğiz. $\bar{\omega}_{\sigma}(f)(p)$ and $\overline{\bar{\omega}}_{\sigma}(f)(p)$ kümeleri de benzer şekilde lineer uzay oldukları gösterilebilir.

$x, y \in\left[\omega_{\sigma}(\mathrm{f})(\mathrm{p})\right]$ ve $\lambda, \mu \in \mathbb{C}$ için $|\lambda|^{P_{m}} \leq k_{1}=\max \left(1,|\lambda|^{H}\right)$ ve $|\mu|^{P_{m}}=k_{2}=\max \left(1,|\mu|^{H}\right)$ olmak üzere

$\left|X_{\sigma(n)}+Y_{\sigma(n)}\right|^{P_{m}} \leq k\left(\left|X_{\sigma(n)}\right|^{P_{m}}+\left|Y_{\sigma(n)}\right|^{P_{m}}\right)$ olduğundan

$\frac{1}{k+1} \sum_{\mathrm{m}=0}^{\mathrm{k}} f\left|\left(\left.\mathrm{t}_{\mathrm{mn}}(\lambda \cdot x+\mu y)\right|^{P_{m}}\right) \leq \mathrm{k} \cdot k_{1} \frac{1}{k+1} \sum_{\mathrm{m}=0}^{\mathrm{k}} f\right|\left(\left.\mathrm{t}_{\mathrm{mn}}(x)\right|^{P_{m}}\right)+\mathrm{k} \cdot k_{2} \frac{1}{k+1} \sum_{\mathrm{m}=0}^{\mathrm{k}} f \mid\left(\left.\mathrm{t}_{\mathrm{mn}}(y)\right|^{P_{m}}\right)$ 
elde edilir (Maddox, 1979).

$\left[\omega_{\sigma}(\mathrm{f})(\mathrm{p})\right]-\lim =0$ ve $x, y \in\left[\omega_{\sigma}(\mathrm{f})(\mathrm{p})\right]$ olduğundan $\lambda \cdot x+\mu y \in\left[\omega_{\sigma}(\mathrm{f})(\mathrm{p})\right]$ sonucu elde edilir. Bu da $\left[\omega_{\sigma}(\mathrm{f})(\mathrm{p})\right]$ nin skaler çarpımla vektörel toplamlı bir lineer uzay olduğunu verir (Kara, 1994).

\section{Materyal ve Yöntem}

\section{Teorem 2.}

$P=\left(P_{m}\right)$ dizisi her $\mathrm{m}$ için $P_{m}>0, \operatorname{Sup}_{m}<\infty$ olsun. Bu takdirde

$\left[\omega_{\sigma}(\mathrm{f})(\mathrm{p})\right], M=\max \left(1, \quad \operatorname{Sup} P_{m}\right)$ olmak üzere

$$
g\left(f(x)=\operatorname{Sup}_{\mathrm{k}, \mathrm{n}}\left(\frac{1}{k+1} \sum_{\mathrm{m}=1}^{k}\left(\left|\mathrm{t}_{\mathrm{mn}} f(\mathrm{x})\right|^{P_{m}}\right)\right)^{\frac{1}{m}}\right.
$$

Fonksiyonu ile tam paranorumlu bir lineer topolojik uzaydır.

\section{İspat.}

Teoremin ispatı için $g:\left[\omega_{\sigma}(f)(p)\right] \rightarrow \mathbb{C}$ ye bir fonksiyon ve $x \in\left[\omega_{\sigma}(f)(p)\right]$ için $\operatorname{Sup}_{\mathrm{k}, \mathrm{n}}\left(\frac{1}{k+1} \sum_{\mathrm{m}=0}^{k}\left(\left|\mathrm{t}_{\mathrm{mn}} f(\mathrm{x})\right|^{P_{k}}\right)\right)<\infty$ olduğundan $g(f(x), \mathbb{C}$ de tanımlıdır. Şimdi paranorm şartlarını sağlatalım.

$$
\begin{aligned}
\text { i) } \quad g\left(f(0)=\operatorname{Sup}_{\mathrm{k}, \mathrm{n}}\left(\frac{1}{k+1} \sum_{\mathrm{m}=0}^{k}\left(\left|\mathrm{t}_{\mathrm{mn}} f(0)\right|^{P_{m}}\right)\right)^{\frac{1}{m}}=0\right. \text { olur } \\
\text { ii) } g\left(f(-x)=\operatorname{Sup}_{\mathrm{k}, \mathrm{n}}\left(\frac{1}{k+1} \sum_{\mathrm{m}=0}^{k}\left(\left|\mathrm{t}_{\mathrm{mn}} f(-\mathrm{x})\right|^{P_{m}}\right)\right)^{\frac{1}{m}}\right. \\
=\operatorname{Sup}_{\mathrm{k}, \mathrm{n}}\left(\frac{1}{k+1} \sum_{\mathrm{m}=0}^{k}\left(\left|\mathrm{t}_{\mathrm{mn}} f(-1 . \mathrm{x})\right|^{P_{m}}\right)\right)^{\frac{1}{m}} \\
=\operatorname{Sup}_{\mathrm{k}, \mathrm{n}}\left(\frac{1}{k+1} \sum_{\mathrm{m}=1}^{k}\left(\left|\mathrm{t}_{\mathrm{mn}} f(|-1||\mathrm{x}|)\right|^{P_{m}}\right)\right)^{\frac{1}{m}} \\
=\operatorname{Sup}_{\mathrm{k}, \mathrm{n}}\left(\frac{1}{k+1} \sum_{\mathrm{m}=1}^{k}\left(\left|\mathrm{t}_{\mathrm{mn}} f(\mathrm{x})\right|^{P_{m}}\right)\right)^{\frac{1}{m}} \\
=g(f(x)
\end{aligned}
$$

olur (Mursaleen, 1983).

iii) $m \geq 1$ ve $\forall m$ için $\frac{P_{m}}{M} \leq 1$ olduğundan

$$
\begin{aligned}
& g\left(f(x+y)=\operatorname{Sup}_{\mathrm{k}, \mathrm{n}}\left(\sum_{\mathrm{m}=0}^{k}\left(\left|\mathrm{t}_{\mathrm{mn}} f(\mathrm{x}+\mathrm{y})\right|^{P_{m}}\right)\right)^{\frac{1}{m}}\right. \\
& \leq \operatorname{Sup}_{\mathrm{k}, \mathrm{n}}\left(\frac{1}{k+1} \sum_{\mathrm{m}=0}^{k}\left(\left|\mathrm{t}_{\mathrm{mn}} f(\mathrm{x})+\mathrm{t}_{\mathrm{mn}} f(\mathrm{y})\right|^{\frac{P_{m}}{M}}\right)^{m}\right)^{\frac{1}{m}}
\end{aligned}
$$




$$
\leq \operatorname{Sup}_{\mathrm{k}, \mathrm{n}}\left(\frac{1}{k+1} \sum_{\mathrm{m}=0}^{k}\left(\left|\mathrm{t}_{\mathrm{mn}} f(\mathrm{x})\right|^{\frac{P_{m}}{M}}\right)^{m}\right)^{\frac{1}{m}}+\operatorname{Sup}_{\mathrm{k}, \mathrm{n}}\left(\frac{1}{k+1} \sum_{\mathrm{m}=0}^{k}\left(\left|\mathrm{t}_{\mathrm{mn}} f(\mathrm{y})\right|^{\frac{P_{m}}{M}}\right)^{m}\right)^{\frac{1}{m}}
$$

$\leq \operatorname{Sup}_{\mathrm{k}, \mathrm{n}}\left(\frac{1}{k+1} \sum_{\mathrm{m}=0}^{k}\left(\left|\mathrm{t}_{\mathrm{mn}} f(\mathrm{x})\right|^{P_{k}}\right)\right)^{\frac{1}{m}}+\operatorname{Sup}_{\mathrm{k}, \mathrm{n}}\left(\frac{1}{k+1} \sum_{\mathrm{m}=0}^{k}\left(\left|\mathrm{t}_{\mathrm{mn}} f(\mathrm{y})\right|^{P_{k}}\right)\right)^{\frac{1}{m}}$

$=g(f(x)+g(f(y)$

olur (Oğur, 2020).

Şimdi $\left[\omega_{\sigma}(\mathrm{f})(\mathrm{p})\right]$ deki skaler çarpımın sürekliliğini gösterelim. $P=\left(P_{m}\right)$ dizisi için $\operatorname{Sup} P_{m}<$ $\infty$ olduğundan $P_{m}>\delta$ olacak şekilde bir $\delta>0$ sayısı vardır.

Şimdi $|\lambda| \leq 1$ için $|\lambda|^{P_{m}} \leq|\lambda|^{\delta}$ olduğundan

$$
\begin{aligned}
g(f(x) & =\operatorname{Sup}_{\mathrm{k}, \mathrm{n}}\left(\frac{1}{k+1} \sum_{\mathrm{m}=0}^{k}\left(\left|\mathrm{t}_{\mathrm{mn}} f(\lambda \cdot x)\right|^{P_{m}}\right)\right)^{\frac{1}{m}} \\
& \leq|\lambda|^{\frac{\delta}{m}} \cdot g(f(x)
\end{aligned}
$$

olur. Böylece $\lambda \rightarrow 0, x \rightarrow 0$ ise $\lambda x \rightarrow 0$ ve eğer $\lambda$ sabit ve $x \rightarrow 0$ ise $\lambda x \rightarrow 0$ dir.

$x \in\left[\omega_{\sigma}(\mathrm{f})(\mathrm{p})\right]$ sabit ve verilen $\varepsilon>0$ için $\exists k_{0}$ sayısı vardır ki $\forall n i c ̧ i n$

$$
\operatorname{Sup}_{\mathrm{k} \geq k_{0}}\left(\frac{1}{k+1} \sum_{\mathrm{m}=0}^{k}\left(\left|\mathrm{t}_{\mathrm{mn}} f(\lambda \cdot x)\right|^{P_{m}}\right)\right)^{\frac{1}{m}}<\frac{\varepsilon}{2}
$$

ve $|\lambda|<\delta$ olacak şekilde $\delta>0$ sayısı bulabiliriz (Nakano, 1953).

dolayısıyla $\forall n$ için

$$
\operatorname{Sup}_{\mathrm{k} \leq k_{0}}\left(\frac{1}{k+1} \sum_{\mathrm{m}=0}^{k}\left(\left|\mathrm{t}_{\mathrm{mn}} f(\lambda \cdot x)\right|^{P_{m}}\right)\right)^{\frac{1}{m}}<\frac{\varepsilon}{2}
$$

olur. Böylece (3) ve (4) ifadelerinden $|\lambda|<\delta$ oldukça $g(f(\lambda . x))<\varepsilon$ elde ederiz ki bu da ispat1 tamamlar (Savaş, 2018).

\section{Bulgular}

$\left[\omega_{\sigma}(f)(p)\right]$ uzayının g paranormuna göre tam olduğu görülecektir.

$\left(x^{s}\right) \operatorname{nin}\left[\omega_{\sigma}(\mathrm{f})(\mathrm{p})\right]$ de bir Cauchy dizisi olsun. Yani

$s, t \rightarrow \infty$ iken $g\left(f\left(x^{s}-x^{t}\right) \rightarrow 0\right.$ olsun.

$g\left(f\left(x^{s}-x^{t}\right)=\operatorname{Sup}_{\mathrm{k}, \mathrm{n}}\left(\frac{1}{k+1} \sum_{\mathrm{m}=0}^{k}\left(\left|\mathrm{t}_{\mathrm{mn}} f\left(x^{s}-x^{t}\right)\right|^{P_{m}}\right)\right)^{\frac{1}{m}}\right.$

ve

olduğundan $\forall m$ ve $n i c ̧ i n$

$$
g\left(f\left(x^{s}-x^{t}\right) \geq \operatorname{Sup}_{\mathrm{k}, \mathrm{n}}\left(\frac{1}{k+1} \sum_{\mathrm{m}=0}^{k}\left(\left|\mathrm{t}_{\mathrm{mn}} f\left(x^{s}-x^{t}\right)\right|^{P_{m}}\right)\right)^{\frac{1}{m}}\right.
$$

$\lim _{s, t \rightarrow \infty}\left|t_{\mathrm{mn}} f\left(x^{s}-x^{t}\right)\right|^{P_{m}}=0$

elde edilir. Özel olarak $\mathrm{m}=0$ olmak üzere herhangi $\mathrm{n}$ için $s, t \rightarrow \infty$ iken 
$\left|\mathrm{t}_{\mathrm{mn}} f\left(x^{s}-x^{t}\right)\right|=f\left(\left|x^{s}-x^{t}\right|\right)=\left|x^{S}-x^{t}\right| \rightarrow 0$ dir. Böylece $\left(x^{s}\right), \mathbb{C}$ de bir Cauchy dizisidir.

$\mathbb{C}$ tam olduğundan dolayı $s \rightarrow \infty$ iken $x^{S} \rightarrow x$ olacak şekilde $x \in \mathbb{C}$ vardır. (5) numaralı ifadeden $\varepsilon>0$ ve $\exists \mathbb{N}$ öyleki $s, t>\mathbb{N}$ için

olur.

$$
\left(\frac{1}{k+1} \sum_{\mathrm{m}=0}^{k}\left(\left|\mathrm{t}_{\mathrm{mn}} f\left(x^{s}-x^{t}\right)\right|^{P_{m}}\right)\right)^{\frac{1}{m}}<\varepsilon
$$

Şimdi $s>\mathbb{N}$ ve $t \rightarrow \infty$ olsun. Bu takdirde (6) numaralı ifadeden

$\left(\frac{1}{k+1} \sum_{\mathrm{m}=0}^{k}\left(\left|\mathrm{t}_{\mathrm{mn}} f\left(x^{s}-x^{t}\right)\right|^{P_{m}}\right)\right)^{\frac{1}{m}}<\varepsilon$ elde edilir. Buradan da

$\delta \rightarrow \infty$ iken $g\left(f\left(x^{s}-x^{t}\right) \rightarrow \infty\right.$ olur.

Bu da $x^{s} \rightarrow x^{\prime} e$ yakınsaması demektir (Mursaleen, 1983).

(6) numaralı ifadeden ve her bir s için $x^{s} \in\left[\omega_{\sigma}(\mathrm{f})(\mathrm{p})\right]$ ve $\left[\omega_{\sigma}(\mathrm{f})(\mathrm{p})\right]$ nin lineer olduğundan $g(f(x))=g\left(f\left(x^{s}-x^{t}+x\right) \leq g\left(f\left(x^{s}-x\right)+g(f(x)) \rightarrow 0\right.\right.$ elde edilir. Buradan $x \in\left[\omega_{\sigma}(\mathrm{f})(\mathrm{p})\right]$ olduğu elde edilir. Böylece $\left[\omega_{\sigma}(\mathrm{f})(\mathrm{p})\right]$ nin tam olduğu görülür (Rafeiro ve ark, 2018).

$\mathrm{Bu}$ teoremde $p$ sabit ve $p \geq 1$ ise $\left[\omega_{\sigma}(\mathrm{f})(\mathrm{p})\right]$ uzayı Banach uzayı $0<p<1$ ise $\mathrm{p}$ - normlu uzaya indirgenir (Sahoo, 1992).

\section{Tartışma ve Sonuç}

\section{Teorem 3.}

$$
P=\left(P_{m}\right) \text { dizisi her } m \text { için } P_{m}>0, \operatorname{Sup} P_{m}<\infty \text { olsun. Bu takdirde }
$$

$\bar{\omega}_{\sigma}(\mathrm{f})(\mathrm{p})$ ve $\overline{\bar{\omega}}_{\sigma}(\mathrm{f})(\mathrm{p})$ uzayları $M=\max \left(1, \quad S u p P_{m}\right)$ olmak üzere

$g\left(f(x)=\operatorname{Sup}_{\mathrm{n}}\left(\sum_{\mathrm{m}=0}^{\infty} f\left(\left|\Psi_{\mathrm{mn}}-\Psi_{\mathrm{m}-1, \mathrm{n}}\right|^{P_{m}}\right)\right)^{\frac{1}{m}}\right.$ fonksiyonu altında bir tam paranormlu lineer topolojik uzaydır.

Teoremin ispatı teorem 2'nin ispatının benzeri olduğundan tekrar vermeyeceğiz.

\section{Teorem 4.}

a) Eğer $\forall m$ için $p_{m} \leq q_{m}$ ise $\bar{\omega}_{\sigma}(\mathrm{f})(\mathrm{p}) \subset \bar{\omega}_{\sigma}(\mathrm{f})(\mathrm{q})$ ve $\overline{\bar{\omega}}_{\sigma}(\mathrm{f})(\mathrm{p}) \subset \overline{\bar{\omega}}_{\sigma}(\mathrm{f})(\mathrm{q})$ dur.

b) Eğer $p$ sabit ve $p \geq 1$ ise

$$
l_{p}^{\sigma}(f)_{p} \subset \bar{\omega}_{\sigma}(\mathrm{f})_{p} \text { ve } l_{\sigma}^{\sigma \sigma}(f)_{p} \subset \overline{\bar{\omega}}_{\sigma}(\mathrm{f})_{p} \operatorname{dir} .
$$

\section{İspat.}

a) $\bar{\omega}_{\sigma}(f)(p) \subset \bar{\omega}_{\sigma}(f)(q)$ göstereceğiz $\overline{\bar{\omega}}_{\sigma}(f)(p) \subset \overline{\bar{\omega}}_{\sigma}(f)(q)$ olduğu benzer şekilde gösterilir.

$x \in \bar{\omega}_{\sigma}(\mathrm{f})(\mathrm{p})$ olsun. Bu takdirde

$\sum_{\mathrm{m}=\mathrm{M}}^{\infty} f\left(\left|\Psi_{\mathrm{mn}}(x)-\Psi_{\mathrm{m}-1, \mathrm{n}}(x)\right|^{P_{m}}\right)<1$

olacak şekilde $M$ tamsayısı vardır. Böylece $\forall n$ ve $m \geq M$ için $f\left(\left|\Psi_{\mathrm{mn}}(x)-\Psi_{\mathrm{m}-1, \mathrm{n}}(x)\right|^{P_{m}}\right)<1$

olup $p_{m} \leq q_{m}$ olduğundan da $f\left(\left|\Psi_{\mathrm{mn}}(x)-\Psi_{\mathrm{m}-1, \mathrm{n}}(x)\right|^{q_{m}}\right)<f\left(\left|\Psi_{\mathrm{mn}}(x)-\Psi_{\mathrm{m}-1, \mathrm{n}}(x)\right|^{P_{m}}\right)$ 
yazılır. Bu eşitliğin her iki tarafının m üzerinden toplamı alınırsa

$\sum_{\mathrm{m}=\mathrm{M}}^{\infty} f\left(\left|\Psi_{\mathrm{mn}}(x)-\Psi_{\mathrm{m}-1, \mathrm{n}}(x)\right|^{q_{m}}\right) \leq \sum_{\mathrm{m}=\mathrm{M}}^{\infty} f\left(\left|\Psi_{\mathrm{mn}}(x)-\Psi_{\mathrm{m}-1, \mathrm{n}}(x)\right|^{P_{m}}\right)$ elde edilir. Sağdaki seri $\mathrm{n}$ ye göre düzgün yakınsak olduğundan, soldaki seri de $\mathrm{n}$ ye göre düzgün yakınsak olur. $\mathrm{O}$ halde $\bar{\omega}_{\sigma}(\mathrm{f})(\mathrm{p}) \subset \bar{\omega}_{\sigma}(\mathrm{f})(\mathrm{q})$ dur.

b) $l_{p}^{\sigma}(f)_{p} \subset \bar{\omega}_{\sigma}(\mathrm{f})_{p}$ ispatını yapacağız. $l_{\sigma}^{\sigma \sigma}(p) \subset \overline{\bar{\omega}}_{\sigma}(\mathrm{f})_{p}$ olduğu da benzer şekilde gösterilir.b) $x \in l_{p}^{\sigma}(f)_{p}$ olsun $p=1$ iken ifade açıtır. $p>1$ için

$$
\begin{gathered}
f\left(\left|\Psi_{\mathrm{kn}}(x)-\Psi_{\mathrm{k}-1, \mathrm{n}}(x)\right|^{p}\right) \leq \frac{1}{k(k+1)^{p}} \sum_{\mathrm{m}=1}^{\infty} m^{p} f\left(\left|\mathrm{t}_{\mathrm{mn}}(x)-\mathrm{t}_{\mathrm{m}-1, \mathrm{n}}(x)\right|^{p}\right) \\
\leq \sum_{\mathrm{m}=1}^{\infty} m^{p} f\left(\left|\mathrm{t}_{\mathrm{mn}}(x)-\mathrm{t}_{\mathrm{m}-1, \mathrm{n}}(x)\right|^{p}\right) \sum_{\mathrm{k}=\mathrm{m}}^{\infty} \frac{1}{k(k+1)^{p}} \\
\leq \sum_{\mathrm{k}=\mathrm{m}}^{\infty} m^{p} f\left(\left|\mathrm{t}_{\mathrm{mn}}(x)-\mathrm{t}_{\mathrm{m}-1, \mathrm{n}}(x)\right|^{p}\right)
\end{gathered}
$$

elde edilir. (II) yakınsak olduğundan $\sum_{\mathrm{m}=1}^{\infty} f\left(\left|\Psi_{\mathrm{mn}}(x)-\Psi_{\mathrm{m}-1, \mathrm{n}}(x)\right|^{p}\right)$ düzgün yakınsaktır. O halde $l_{p}^{\sigma}(f)_{p} \subset \bar{\omega}_{\sigma}(\mathrm{f})_{p}$ olur (Savaş, 2018).

Sonuç olarak modülüs fonksiyon yardımı ile tanımlanan invaryant yakınsak dizi uzayları tanımlanarak bazı kapsamlar kurulmuştur.

$\left[\omega_{\sigma}(f)\right], \bar{\omega}_{\sigma}(f)$ ve $\overline{\bar{\omega}}_{\sigma}(f)$ uzayları $\left[\omega_{\sigma}(f)(p)\right], \bar{\omega}_{\sigma}(f)(p)$ ve $\overline{\bar{\omega}}_{\sigma}(f)(p)$ uzaylarına genişletilerek genel dizi uzaylarının topolojik özellikleri incelenmiştir.

\section{Kaynakça}

Kara, H. (1994). İnvaryant yakınsaklık yardımıyla tanımlanan dizi uzayları. (Doktora Tezi), Yüzüncü Y1l Üniversitesi, Fen Bilimleri Enstitüsü, Van, Türkiye.

Lorentz, G. (1948). A contribution to the theory of divergent secunces. Acta Mathematica. 80, 167-190. doi: 10.1007/BF02393644.

Maddox, I. J. (1979). On strong alost convergence. Mathematical Proceedings of the Cambridge Philosophical Society. 85, 345-350. doi:10. 1017/S0305004100054281.

Mursaleen, M. (1983). On some new invariant matrix methods of summability. Quarterly Journal of Mathematics, 34, 77. doi:10.1093/qmath/34.1.77.

Nakano, H. (1953). Concave modulars. Journal of the Mathematical Society of Japan, S:29-49. doi:10.2969/jmsj/00510029.

Oğur, O. (2020). Modülüs fonksiyonu ile tanımlanmış genelleştirilmiş büyük lebesgue dizi uzaylarının topolojik bazı özellikleri. Gümüşhane Üniversitesi Fen Bilimleri Enstitüsü Dergisi 10 (4), 11481149. do1: 10.17714 /gumusfenbil.732116.

Rafeiro, H., Samkho, S. \& Umarkhadzhiev, S. (2018). Grand lebesgue sequence spaces. Georgian Mathematical Journal, 19(2), 235-246. doi:org/10.1515/gmj-2018-0017.

Sahoo, G. D. (1992). On some squence spaces. Journal of Mathematical Analysis and Aplications 164, 381-398. doi:10.1016/0022-247X(92)90122-T.

Savaş, E. (2018). On some new sequence spaces. Journal of the Institute of Science and Technology of Balıkesir University. Special Issue, 20(3). 155-156. doi: 10.25092/baunfbed.487747. 